\title{
Entitativity and Implicit Measures of Social Cognition
}

\author{
Ben Phillips \\ bsphilli@asu.edu
}

Forthcoming in Mind \& Language

\begin{abstract}
I argue that in addressing worries about the validity and reliability of implicit measures of social cognition, theorists should draw on research concerning "entitativity perception." In brief, an aggregate of people is perceived as highly "entitative" when its members exhibit a certain sort of unity. For example, think of the difference between the aggregate of people waiting in line at a bank versus a tight-knit group of friends: the latter seems more "groupy" than the former. I start by arguing that entitativity perception modulates the activation of implicit biases and stereotypes. I then argue that recognizing this modulatory role will help researchers to address concerns surrounding the validity and reliability of implicit measures.
\end{abstract}

\section{Introduction}

Consider the stark difference between a North Korean military parade, and the people wandering around Times Square on a busy day. The collection of people marching in the parade is unified in various ways: picture the perfectly synchronized goose-stepping; the matching uniforms; and the shared sense of national pride. In contrast, the aggregate of people in Times Square is relatively disunified: people are walking in different directions; they don't know each other; and they are there for different reasons. There is some sense in which the former has the property of being a group to a greater extent than the latter. 
In 1958, Campbell tried to capture this intuitive difference by coining the somewhat awkward term, "entitativity." He defined it as the degree to which a collection of people have "the nature of an entity" $(1958,17)$. In addition to outlining a metaphysics of entitativity, Campbell also sketched a psychological theory. Drawing on Gestalt principles of perceptual organization, he suggested that a group is perceived as highly entitative when its members stand in certain relations to one another, such as being in close proximity; being similar; and moving together in accordance with shared goals.

While Campbell's claims were plausible enough, he did not support them with evidence. In fact, his ideas lay dormant until the turn of the century, when social psychologists began to carry out experimental work on entitativity perception. Since then, numerous studies have found that when we perceive a group, we represent its degree of entitativity (for a review, see Hamilton, 2007). Various studies have also found that entitativity perception is a key precursor to bias and stereotyping. For example, in Crawford and colleagues' (2002) study, participants observed the member of a group as exhibiting some trait (e.g. being lazy). They were then tested on whether they associate this trait with other members of the group. Crawford and colleagues found that participants were much more likely to do so when they perceived the group as highly entitative.

By the time empirical work on entitativity perception had taken off, studies of implicit bias and implicit stereotyping were well under way. Social psychologists have developed a number of implicit measures, such as the Implicit Association Test (Greenwald et al., 1998); the Go/No-Go Association Task (Nosek \& Banaji, 2001); the Weapon Identification Task (Payne, 2001); the Affect Misattribution Procedure (Payne, 2009); the Bona Fide Pipeline (Fazio et al., 1995); and the Affective Lexical Priming Score (Lebrecht et al., 2009). 
It is important to note that theorists use the term "implicit" in various ways. Some use it to denote characteristics of the constructs being measured. For example, some use the term to denote states that are automatically activated in a way that is inaccessible to introspection (e.g., see Greenwald et al., 1998). Others use the term to characterize those measurement procedures that are designed to detect biases and stereotypes without relying on self-report (e.g., see De Houwer \& Moors, 2010). In what follows, I focus on problems surrounding implicit measures of biases and stereotypes. In doing so, I remain as neutral as possible regarding the nature of the biases and stereotypes being measured (e.g. I do not take a stance on whether they are activated in a way that is inaccessible to introspection).

A number of meta-analyses have found that implicit measures of biases and stereotypes tend to have weak validity and reliability (e.g., see Bar-Anan \& Nosek, 2014; Forscher et al., 2019; Oswald et al., 2013). Some theorists have reacted by questioning the utility of these measures. For example, commenting on the Implicit Association Test, Singal (2017) implores social psychologists to "reexamine their decision to devote so much time and energy to this one instrument." Others have suggested that instead of turning their attention away from implicit measures of bias and stereotyping, social psychologists should find ways to improve their validity and reliability (e.g., see Cooley \& Payne, 2017; Gschwendner et al., 2008). In what follows, I pursue this second approach. I argue that to improve the validity and reliability of implicit measures, social psychologists should draw on research into entitativity perception.

More specifically, in section 2, I present evidence that entitativity perception modulates the activation of implicit biases and stereotypes. In sections 3 and 4, I address concerns surrounding the validity and reliability of implicit measures. I start by arguing that implicit biases and stereotypes are cross-contextually unstable, in large part, because entitativity perception is 
context-sensitive. I then argue that the validity and reliability of implicit measures will improve if researchers take this source of cross-contextual instability into account. Finally, in section 5, I outline an account of how entitativity perception modulates the activation of implicit biases and stereotypes.

\section{Entitativity perception as a precursor to implicit bias and stereotyping}

Multiple studies have converged on the view that we really do perceive some groups as more entitative than others. In what follows, I review the evidence. I then present additional evidence that entitativity perception is a precursor to implicit bias and implicit stereotyping.

\subsection{A folk typology of groups}

In a foundational series of studies, Lickel and colleagues (2000) sought to uncover the features that are most predictive of entitativity perception. Participants rated the degree to which various collections of people "qualify as a group" (e.g. a family, women, people in line at the bank, etc.). Then, in a second study, participants rated these groups on various aspects, such as the similarity of its members; the sharing of common goals; and degree of interaction among group members. There were two major findings.

First, the groups clustered around 5 basic types: (1) intimacy groups (e.g. friendship groups, families); (2) task groups (e.g. committees, sporting teams); (3) social categories (e.g. women, doctors); (4) loose associations (e.g. people working at the same large factory); and (5) transitory groups (e.g. people waiting at a bus stop). Lickel and colleagues' second major finding was that a clear entitativity continuum emerged. Intimacy groups were seen as the most entitative;

followed by task groups; then social categories; and so on for each successive member in the list above. 
Several studies have replicated these initial findings (for an overview, see Hamilton, 2007). In fact, Sherman and colleagues (2002) found that people spontaneously subsume groups under the superordinate categories listed above. Additional studies have also found that high-entitative groups are not processed in the same way as low-entitative groups. For example, the former are processed in a more on-line and "integrative" manner (Hamilton et al. 2002); they are seen as more of a threat than low-entitative groups (e.g., see Fessler \& Holbrook, 2016); they are seen as having more agency than low-entitative groups (Tanibe et al., 2019); and they are seen as having greater collective responsibility for the actions of individual members (Abelson et al., 1998;

Leonard, 2019; Lickel et al., 2003; Newheiser et al., 2012).

\subsection{Implicit stereotyping}

In addition to all these differences, high-entitative groups are stereotyped more than lowentitative groups. In a seminal study, Crawford and colleagues (2002) deployed a modified version of the savings-in-relearning paradigm: the core assumption being that it is easier to relearn previously learned associations than it is to form brand new ones.

Participants viewed 32 photographs of individuals drawn from two groups. In the exposure task, each photograph was paired with a description of some trait-implicative behavior (e.g. solving a complex mathematical problem). Half of the members of each group were paired with behaviors that implied one trait (e.g. aggressiveness), while the other half were paired with behaviors that implied a different trait (e.g. intelligence). Each group was then described in terms that implied either high or low entitativity. For example, members of low entitative groups were described as having "different opinions," and as acting "in a different manner" across a variety of situations $(2002,1080)$. 
In the paired associates task, the 32 photographs were presented to participants again; however, each photograph was now paired with a trait term. Participants were instructed to remember each photograph-word pairing. Importantly, half of the photographs were paired with a trait term that matched the description given during the exposure task. For example, an individual that was initially described as solving a complex mathematical problem was paired with the term "intelligent." These were referred to as trait inference trials. The other photographs were paired with the trait term that was associated with other members of the relevant group, but not with the individual in question. For example, suppose each member of group A was described as exhibiting either intelligent or aggressive behavior during the exposure task. In that case, if a given group member was described as solving a complex mathematical problem during the exposure task, they were paired with the term "aggressive," not the term "intelligent." These were referred to as trait transference trials.

In the cued-recall task, each photograph was presented to participants again. They were told to write down the word that appeared on the photograph during the paired associates task.

What Crawford and colleagues found was that for trait inference trials, recall of photographword pairs was most accurate for low entitative groups. For example, suppose $S$ was described as solving a complex mathematical problem during the exposure task, before being paired with the term "intelligent" during the paired associates task. In that case, participants were better at remembering the latter pairing when $S$ was the member of a low entitative group.

In contrast, for trait transference trials, recall of photograph-word pairs was most accurate for high entitative groups. For example, suppose the description of $S$ 's behavior during the initial exposure task did not imply laziness; whereas, the description of fellow-group member $T$ 's behavior did imply laziness. Moreover, suppose $S$ 's photograph was paired with the word "lazy" 
during the paired associates task. In that case, participants were better at remembering the pair, $<S$, lazy $>$, when the group $S$ and $T$ both belonged to was highly entitative.

Overall, this pattern of results provides evidence that perceiving a group as highly entitative leads to the stereotyping of its members (for similar findings, see Spencer-Rodgers et al., 2007; Susskind et al., 1999; Yzerbyt et al., 1998). According to Crawford and colleagues' model, the members of those groups that are perceived as highly entitative are seen as interchangeable. Thus, a group impression is formed based on the trait-implying behaviors of individual members. This impression is then spontaneously transferred to others in the group, even if they have not been observed exhibiting a behavior that is indicative of this trait. In contrast, for groups that are perceived as low in entitativity, members are processed as individuals. Thus, if one member is seen as exhibiting a trait-implying behavior, the trait in question does not become associated with other group members.

\subsection{Implicit bias}

Other experimental paradigms provide evidence that people tend to harbor implicit biases against highly entitative groups. Gaertner and colleagues (2008) told people that they were participating in an experiment about people's reactions to noise exposure. Participants sat next to two confederates and filled out a form, before a third confederate entered the room. In the lowentitativity condition, the two seated confederates remained silent. In the high-entitativity condition, one of them acknowledged the third confederate, and remarked on a volleyball game, saying, "We weren't sure if you would make the game." The third confederate replied, "I would not miss a game." To further enhance perceived entitativity, the confederates also wore the same shirt in this condition: it had the words "Bryan-College-Station Volleyball: Team United" printed on it. The experimenter then returned to announce that a mistake had been made, and that they 
only needed three participants. In the rejection condition, one of the confederates glared at the participant and said scornfully, "S/he should be the one that leaves!" In the no-rejection condition, the participant was excluded via the drawing of cards, which were rigged to ensure rejection.

In one study, participants were then invited to fill in for an absent experimenter. The participants' job was to control the noise level to which the other "participants" were exposed. They were told that, when prompted, they should adjust the volume to a level anywhere between 0 to $110 \mathrm{~dB}$, where $110 \mathrm{~dB}$ would cause "significant discomfort." Having chosen a volume, participants then rated the degree to which the other "participants" constituted a group.

Gaertner and colleagues found that the mean volume selected by participants was the highest when they were rejected by, what they perceived to be, a highly entitative group. Interestingly, the mean volume selected by participants was the lowest when they were rejected by, what they perceived to be, a low entitative group; while the mean volumes selected in the two no-rejection conditions were somewhere in between. Gaertner and colleagues conclude that when participants are rejected by the member of a group that they perceive as highly entitative, they associate rejection with the group itself, and retaliate accordingly.

In a second study, the same set-up was used; however, rather than filling in for an absent experimenter, participants were asked to complete a version of the Implicit Association Test (IAT). The IAT is designed to measure the strength of association between social concepts (e.g. $B L A C K$ or WHITE) and valenced concepts (e.g. PLEASANT or UNPLEASANT). In Gaertner and colleagues' version of the IAT, participants saw words and photographs on a computer screen. The instructions indicated that all and only those people wearing the shirt with "Team United" printed on it are members of Team United. In a series of trials, participants were tasked with 
categorizing the words as either good or bad, and the photographed individuals as either Team United members or not Team United members. As in the first study, the participants were also asked to rate the degree to which the other "participants" in the study constituted a group.

Gaertner and colleagues found that those participants who were rejected by a member of Team United during the first phase of the study exhibited the least favorable associations with Team United. For example, these participants were the most likely to associate the words "horrible" and "nasty" with members of Team United. Participants who were either not rejected, or were rejected by what they perceived to be a low entitative group, were less likely to associate negative words with members of Team United.

\section{Validity, reliability, and concept accessibility}

I have provided evidence that entitativity perception modulates the encoding and activation of implicit biases and stereotypes. In what follows, I argue that by further examining this role, we can address some of the main concerns surrounding the validity and reliability of implicit measures.

\subsection{Validity and reliability}

Various meta-analyses have found that, on average, implicit measures have weak predictive validity: that is, they are relatively poor predictors of behavior (e.g., see Forscher et al., 2019; Oswald et al., 2013). Other meta-analyses have found that, on average, implicit measures have low test-retest reliability: that is, individual's scores vary considerably over time (e.g., see BarAnan \& Nosek, 2014; Gawronski et al., 2017).

In addressing these concerns, a number of theorists have turned their attention to the contextsensitive manner in which implicit attitudes are expressed (see Barden et al., 2004; Brownstein et al., 2020; Cooley \& Payne, 2017; Gschwendner et al., 2008; Jost, 2019; Payne et al., 2017). For 
example, Cooley and Payne (2017) found that both the predictive validity and test-retest reliability of the Affective Misattribution Procedure were significantly enhanced when images of social groups were used instead of images depicting a lone member. They argue that an image of a group activates relevant biases and stereotypes more effectively than an image of a lone group member, because the former is seen as more typical of the given social category $(2016,48)$. Moreover, relative to groups, individuals belong to myriad social categories. Thus, when observing an individual, which stereotypes and biases are activated will depend on which social categories are salient; and this, in turn, will fluctuate with the subject's temporary mood, goals, and immediately prior experiences $(2016,46)$.

In a similar vein, Gschwendner et al. (2008) gave German participants a version of the IAT, in which they were exposed to images of both German and Turkish faces. In the experimental condition, there was an image of a mosque in the background; whereas, in the control condition, there was an image of a garden. The stability of participants' scores was assessed over a two week period. Participants in the control condition exhibited a low stability coefficient of 0.29 , whereas participants in the experimental condition exhibited a high stability coefficient of 0.72 . Gschwendner et al. (2008) conclude that the image of the mosque enhanced the accessibility of the concept, TURKISH, and thereby enhanced the accessibility of any biases participants harbored against Turkish people. More generally, they suggest that the reliability of implicit measures will be stronger when (i) repeated measurements are taken in the same context, and (ii) the context is such that the construct being measured is highly accessible, i.e. it is highly poised for use in cognitive processing $(2008,71)$.

In sum, then, the studies outlined above suggest that there are important constraints on the activation of implicit biases and stereotypes. In what follows, I argue that by drawing on studies 
of entitativity perception, researchers can gain a greater understanding of how these constraints work. More specifically, I will argue that entitativity perception modulates the activation of implicit biases and stereotypes by making the relevant social categories more accessible. However, entitativity perception is context-sensitive. Thus, in designing implicit measures that are valid and reliable, researchers should ensure that entitativity cues are salient and stable.

\subsection{Entitativity perception and top-down salience effects}

Recall the folk typology of groups uncovered by Lickel and colleagues (2000). Intimacy groups are perceived as the most entitative; followed by task groups; then social categories; then loose associations; and finally, transitory groups.

Notice, however, that membership in each group-type is graded. To some degree, my social soccer team counts as a task group (e.g. we want to win games together); but it also counts as an intimacy group to a significant degree too (e.g. we are friends). Similarly, consider a group of people boarding a plane. This counts as a transitory group. However, in lining up, members of the group must coordinate their actions to some degree: the group therefore counts as a task group to a non-negligible degree.

We can therefore think of the different group-types as different dimensions along which groups are seen as varying. A group's position along each dimension will be determined by the degree to which it possesses certain features. For example, Lickel and colleagues (2000) found that people see quality of interaction as determining membership in an intimacy group; whereas, for task groups, they see the sharing of goals as the key determinant. Given that groups vary along these different dimensions_ — possessing these diagnostic features to different degrees—we should expect to observe salience effects. 
For example, suppose you are comparing the average career of a police officer with the average career of a fire fighter. You do so by comparing day-to-day duties, salaries, benefits, and so on. In this context, the salient features of each group are diagnostic of social categories. This means that you are likely to perceive both groups as relatively low in entitativity. ${ }^{1}$ But consider a different context in which you are reading an article about tensions between the police and certain communities (e.g. tensions that have arisen because of police shootings, racial profiling, or the treatment of protestors). In this context, those features that are diagnostic of task and intimacy groups will be more salient. For example, the article you are reading might invoke the so-called "code of silence"; the various goals that police officers share; and the coordinated activities they engage in when dealing with rioters and protesters. Given the salience of these features, you probably see police officers as constituting a more entitative group than you did in the first context.

Direct evidence for the context-sensitivity of entitativity perception comes from a study by Bangerter (2005). Participants were asked to rate the entitativity of various age groups. Importantly, though, Bangerter varied the way in which each age group was labelled. In some cases, generational labels were used (e.g. "Baby Boomers"). In other cases, age-labels were used (e.g. "People who are about 75 years old"). Cohort-labels were also used (e.g. "People born between 1945 and 1950").

There were two key findings. First, age groups were seen as the least entitative when they were labelled as a cohort. For example, the group consisting of Baby Boomers was seen as more entitative when labelled as "Baby Boomers" than it was when labelled as "People born between

\footnotetext{
${ }^{1}$ Below, I consider a different form of entitativity perception, which I refer to as "categorical entitativity perception." There is evidence that some social categories are perceived as high in "categorical entitativity," even if they are not highly entitative in the sense operative above.
} 
1946 and 1964." The second key finding was that labelling had an effect on whether certain features were utilized as entitativity cues. For instance, when a group was labelled in generational terms, common goals and values were predictors of entitativity perception. However, when labelled in terms of age, these same features were not utilized as entitativity cues.

If the way in which an age group is labelled can determine whether certain features are processed as entitativity cues, it is very likely that entitativity perception will vary with other aspects of the context. For example, suppose we are debating whether "Ok, Boomer" should be regarded as a slur. The various tensions that exist between Baby Boomers and younger generations therefore loom large (e.g. tensions surrounding who is responsible for the global financial crisis in 2008; who is responsible for climate inaction; etc.). In this sort of context, the goals and values that Baby Boomers purportedly share will be salient, thereby making this generational group seem more entitative than it does in other contexts (e.g. a context in which we are discussing intergenerational differences in health).

Additional evidence that entitativity perception is context-sensitive comes from studies of racial categorization. Some studies have found that racial groups are perceived as relatively low in entitativity (see Haslam et al., 2000; Lickel et al., 2000). Invariably, though, these studies ask participants to rate the entitativity of different racial groups without putting them into a wider context; rather, the groups usually appear on a list with other groups, e.g. "Women," "Doctors," "Citizens of the US," etc. If, for example, tensions between the members of different racial groups are made salient, we should expect these groups to be seen as more entitative than they are in the sorts of studies just described. 
Support for this hypothesis comes from a study by Pietraszewski (2016). Participants were presented with a series of photographs in which different individuals were depicted as making various statements. Half the depicted individuals were Black; while the other half were White. Some of the individuals' statements indicated that they worked for the same charity; however, membership in the charity cut across racial lines. After completing a 1-minute distractor task, the photographs were presented to participants again; only this time, all of the photographs appeared simultaneously, and there was only one statement shown in the middle of the screen. The participants' task was to indicate who issued the statement in question.

Importantly, two conditions were included. In the prime condition, participants viewed a series of photographs during the distractor task. These photographs depicted racial conflict. The participants were also asked to think of "all the racial conflict and inequality you have been exposed to or are aware of" $(2016,87)$. In the nonprime condition, the distractor task involved naming US state capitals, while viewing a map of the US.

Pietraszewski found that when recalling who said what, participants were far more likely to confuse members of the same racial group in the prime condition than in the nonprime condition. Recall that in the nonprime condition, the only salient alliance was the charity group, which cut across racial lines. According to Pietraszewski, this is evidence that we are predisposed to detect and track those groups that constitute alliances. This explains why members of same racial group were conflated more in the prime condition than they were in the nonprime condition: it is because they were processed as members of the same alliance in the prime condition, not as individuals. In the nonprime condition, members of the same racial group were not processed as members of the same alliance, because the only salient alliance (i.e. the charity group) cut across racial lines. 
For our purposes, the key finding is that in one context, attention to the relevant features can cause people see racial groups as highly entitative; whereas, in a different context, in which attention is not drawn to the relevant features, the same racial groups are not processed as highly entitative.

In sum, the degree to which a group is seen as entitative will vary with the context, and which kinds of features are salient. Moreover, given that most (if not all) groups tend to vary along each of the dimensions identified by Lickel and colleagues (2000), context-sensitivity is probably a ubiquitous feature of social categorization. This has important implications for the validity and reliability of implicit measures. As was explained above, biases and stereotypes are more likely to be activated when the target group is seen as highly entitative. If entitativity perception is sensitive to contextual factors, it follows that the activation of biases and stereotypes is sensitive to these same factors. Thus, there are good reasons for thinking that if researchers make entitativity cues highly salient, the validity and reliability of implicit measures will improve.

\subsubsection{Bottom-up salience effects}

In most of the examples given above, entitativity perception was modulated in a top-down manner. For example, thinking about racial conflict caused people to see racial groups as more entitative than they would otherwise. However, bottom-up factors are also an important source of context-sensitivity.

Phillips and colleagues (2018) examined the automatic processes that are triggered when one's visual field is populated by a group of people. Across multiple studies, participants were presented with sets of four faces. In each trial, an initial set of faces was briefly presented, followed by a set of different faces. In the first study, participants were tasked with specifying which set of faces is more racially diverse. In the second study, the task was to specify which set 
is more diverse in terms of gender. Finally, in the third study, the task was to specify which set is the most hierarchical.

By displaying the faces for only $200 \mathrm{~ms}$ before backward masking them, Phillips and colleagues were able to test the hypothesis that we extract information about groups using ensemble coding. Observers use ensemble-coding to extract the central tendency of a set of objects (e.g. their average speed or color) in a rapid, parallel, manner (Ariely, 2001; Haberman \& Whitney, 2009). Two-hundred milliseconds is too fast for more than a single saccade (Thorpe et al., 1996). Thus, by limiting presentation times to $200 \mathrm{~ms}$, Phillips and colleagues were able to prevent participants from processing the faces in a serial manner. To extract accurate group-level impressions, participants would need to utilize an ensemble-coding mechanism.

Phillips and colleagues found that participants were above chance in detecting the more hierarchical groups, as well as the more diverse groups, even though presentation times prevented serial processing. This constitutes evidence that group-impressions are formed rapidly, based on low-level visual cues.

As Phillips and colleagues $(2014,116)$ point out, there is evidence that groups are perceived as more entitative when they are perceived as homogenous (see Hamilton, 2007). Thus, visually perceiving a group as exhibiting low variability with respect to certain features may enhance perceptions of entitativity. There are various studies that provide independent support for this claim. For example, there is evidence that groups whose members exhibit the same facial expression are perceived as highly entitative (Magee \& Tiedens, 2006). There is also evidence that groups whose members exhibit synchronous movements are perceived as highly entitative (Fessler \& Holbrook, 2016; Ip et al., 2009; Lakens, 2010; Lakens \& Stel, 2011; Wilson \& Gos, 
2019). In all these cases, the impression of entitativity is based on a visible feature that exhibits low variability across the group.

These findings point to another way in which entitativity perception varies across contexts, for as Phillips and colleagues (2018) point out, ensemble coding is only triggered when multiple group members are present in one's visual field. In those contexts in which the relevant cues are not visible, let alone salient, entitativity perception will therefore be dampened.

For instance, in most versions of the IAT, members of the same group are displayed in succession, not at the same time. What's more, they are presented as disembodied 2D faces. Given that entitativity perception is attenuated in these sorts of contexts, the accessibility of the relevant biases and stereotypes will be attenuated too: the result will be implicit measures with weak validity and reliability.

The study by Cooley and Payne (2017) illustrates this point. Recall their finding that when participants observed images depicting multiple members of a social group, rather than just a lone member, this enhanced the validity and reliability of the Affective Misattribution Procedure. Given that entitativity cues were more visually salient when multiple group members were depicted, it is not surprising that the relevant biases became more accessible.

Thus, future studies should directly examine the role that visual cues play in driving entitativity perception across contexts, for the cross-contextual instability of implicit biases and stereotypes is likely explained, in large part, by the varying salience of these cues. Moreover, studies of this sort should focus on the kinds of everyday contexts in which people process groups. For example, in cities with relatively high levels of racial segregation (by neighborhood, school, etc.), same-race individuals are often seen walking together, playing together, greeting 
one another, and so on. ${ }^{2}$ People living in these sorts of cities will see the relevant racial groups as more entitative when these cues are visible to them (for a relevant discussion, see Enos, 2017).

\section{The dynamical/categorical distinction}

In explaining the role that entitativity perception plays in modulating the activation of biases and stereotypes, I have been focusing on the notion of entitativity that falls out of Lickel and colleagues' (2000) pioneering work. Some theorists have argued that we should recognize a second notion of entitativity. In what follows, I review the evidence in favor of this view. I argue that it provides us with additional resources for understanding the conditions under which implicit biases and stereotypes are activated.

\subsection{Two ways of thinking about groups}

In a series of studies, Ip and colleagues (2006) asked participants to watch animations, which depicted creatures on another planet. In one condition, creatures in the same group had the same skin color. In another condition, they had different skin colors; however, they exhibited synchronous movements. Participants rated each groups' degree of entitativity, as well as the degree to which its members shared traits or common goals.

Ip and colleagues found that the perception of physical similarity predicted the perception of entitativity, where this was mediated by the perception of common traits, but not common goals. They also found that the perception of synchronous movement predicted the perception of entitativity, but this was mediated by the perception of common goals, not common traits. Ip and colleagues conclude that there are "two different routes" to entitativity perception.

\footnotetext{
${ }^{2}$ For data on racial segregation in US cities, see https://www.brookings.edu/blog/theavenue/2017/12/08/metro-areas-are-still-racially-segregated/
} 
Rutchick and colleagues (2008) extended these findings by manipulating the way in which participants thought about the unity of a target group. In the categorical condition, they were asked to imagine a set of bees flying around the room. They were then told to think about the individual bees as members of the general category "bees." To reinforce the idea that membership in the category is determined by the possession of certain physical traits, they were shown a taxonomical chart.

In the dynamic condition, participants were asked to think about the individual bees in terms of their role in a functioning beehive. To reinforce the idea that membership in the hive is determined by each bee's contribution to the group's "goals," participants were shown diagrams that illustrated the specific roles of different bees.

Participants then learned about three different groups: a Bhutanese social caste; an American political party; and a group of students in Missouri. In one study, participants were asked to list the three most important things about each group. In a second study, participants were asked to rate the entitativity of each one.

Rutchick and colleagues found that in the categorical condition, participants attended to the degree of physical similarity between group members; whereas, in the dynamic condition, they attended to the degree of interaction among group members. Rutchick and colleagues also found that these differences in attention drove perceptions of entitativity. For example, they found that in the categorical condition, seeing members of the high school group as similar predicted entitativity perception; whereas, in the dynamic condition, seeing members of this group as interactive predicted entitativity perception.

Like Ip and colleagues (2006), Rutchick and colleagues conclude that there are two kinds of entitativity perception, which are based on the two different ways of thinking about groups: 
namely, as "categorical" or as "dynamical" (see also Brewer et al., 2004; Haslam et al., 2000;

Wilder \& Simon, 1998). Call these two forms of entitativity perception, "categorical entitativity perception" and "dynamical entitativity perception."

It makes sense that we tend to reify groups in these two ways. If the members of a group are highly similar, this raises the probability that they share certain underlying traits, which thereby imbues the category in question with inductive potential (see Rothbart \& Taylor, 1992; Yzerbyt et al., 1997). This sort of entitativity perception drives "psychological essentialism": the tendency to construe the members of a homogenous group as sharing a hidden trait or "essence" (see Gelman, 2003). By the same token, if the members of a group exhibit a high degree of synchronous movement, this raises the probability that they shared certain goals or norms, which thereby imbues the given category with inductive potential.

\subsection{Top-down salience effects}

By distinguishing between dynamical and categorical entitativity perception, we can deepen our understanding of the conditions under which biases and stereotypes are activated. To see why, consider a study by Spencer-Rodgers and colleagues (2007). They examined the stereotypes that people harbor about two kinds of groups: task groups and social categories. The former are high in dynamical entitativity, while the latter are often high in categorical entitativity. SpencerRodgers and colleagues found that entitativity perception mediates stereotyping for both types of groups. Interestingly, though, they found that the stereotypes differ in systematic ways.

Compared to participants' stereotypes about task groups, their stereotypes about social categories were richer. Most notably, the latter included more physical attributes (e.g. tanned and blonde for Californians); more typical behaviors (e.g. play basketball for African Americans); and more references to contextual objects (e.g. brief cases for career women). In addition to being less 
rich, participants' stereotypes about task groups tended to focus more on the groups' goals and achievements (e.g. won the world series for New York Yankees).

There are other key differences in the way that stereotyping is modulated by each form of entitativity perception. For example, there is evidence that when a group is perceived as high in entitativity, members are seen as responsible for each other's actions (Gaertner et al., 2008; Lickel et al., 2003; Leonard, 2019; Newheiser et al., 2012). Importantly, though, this sort of effect appears to be modulated by dynamical entitativity perception, not categorical entitativity perception. As some theorists have suggested, a natural explanation of this fact is that dynamical groups are seen as having agency in a way that categorical groups are not (Malle, 2010; Newheiser et al., 2010, p. 935; see also Tanibe et al., 2019).

Some studies have also found that those groups that are perceived as high in dynamical entitativity are perceived as a threat. For example, Fessler and Holbrook (2016) got participants to listen to the footsteps of marching soldiers. When the footsteps were synchronous, participants judged the group of soldiers to be more physically formidable, where this was mediated by dynamical entitativity perception (see also Callahan \& Ledgerwood, 2016; Winegard \& Deaner, 2010). Like those described above, this study suggests that dynamical entitativity perception modulates group impressions in unique ways.

Putting this all together, then, the evidence suggests that implicit biases and stereotypes are driven by both categorical and dynamical entitativity perception; however, these forms of entitativity perception generate different kinds of biases and stereotypes. Given that which type of entitativity perception one engages in varies with the context, it follows that which types of biases and stereotypes are activated will vary with the context too. 
To illustrate, consider racial groups again. If the context is such that one is thinking about racial groups in dynamical terms, one's attention will be guided towards certain features (for further discussion, see Phillips, 2020). For example, perhaps one is thinking about tensions between the police force and the African American community. Given that one is construing African Americans in dynamical terms, this will lead to the encoding and activation of certain stereotypes, such as the stereotypic expectation that African Americans are likely to affiliate with one another; to have similar goals; and to engage in coordinated action. It will also lead to the encoding and activation of specific sorts of biases, such as the tendency to hold the group responsible for the actions of individual members (see Gaertner et al., 2008; Lickel et al., 2003; Leonard, 2019; Newheiser et al., 2012), or the tendency to see the group as a threat (e.g., see Fessler \& Holbrook, 2016).

If, however, the context is such that one has not been primed to think of racial groups in dynamical terms, attention will go to those features that drive categorical entitativity perception. As the work by Spencer-Rodgers and colleagues (2007) suggests, this will lead to the encoding and activation of a richer suite of associations. For example, it may activate stereotypic expectations concerning psycho-behavioral tendencies, such as being aggressive or being athletic.

The developmental evidence provides indirect support for this claim. When exposed to generic language about social groups (e.g. "African Americans are X," “Girls love pink," etc.), both children and adults tend to construe these groups in categorical terms. This leads to stereotypic expectations concerning common psycho-behavioral traits (Rhodes, 2012). However, when young children are not exposed to generic language, they tend to construe social categories in dynamical terms. This leads to stereotypic expectations concerning affiliative behavior. For 
example, Shutts and colleagues (2013) found that 4-year-olds use gender and race to predict friendships; however, they only use these categories to predict similar psycho-behavioral tendencies when primed with generic language. This fits with the view, defended above, according to which stereotypic expectations vary across contexts because which type of entitativity perception one engages in varies across contexts as well.

\subsection{Bottom-up salience effects}

Once again, researchers should also be careful to distinguish between those factors that drive entitativity perception in a top-down manner, and those that drive it in a bottom-up manner. Recall that entitativity perception is enhanced when multiple group members are visible; and when certain features are seen as exhibiting low variability across the group. In the context of the dynamical/categorical distinction, this provides us with additional ways in which to explain the cross-contextual instability of implicit biases. For example, if the members of a group are moving in synchrony (e.g. walking together), this may enhance dynamical entitativity perception. If, however, members of the group are not seen as moving in synchrony, this will dampen dynamical entitativity perception and enhance categorical entitativity perception: observers will thereby devote more attention to physical similarities between group members. In fact, this is exactly what Ip and colleagues (2006) found in the study described above. When group members moved in synchrony, this fueled dynamic entitativity perception; however, when this cue was absent, attention went to similarities in skin color, which fueled categorical entitativity perception.

Thus, in designing implicit measures, researchers should control for both (i) which type of entitativity perception is being cued (dynamical or categorical), and (ii) whether entitativity perception is being cued in a top-down or a bottom-up manner. It is likely that different sorts of 
cues make the relevant biases and stereotypes accessible to different degrees. Examining these differences in accessibility will enable researchers to design implicit measures with more validity and reliability. For example, it is possible that dynamical entitativity perception is strongest when members of the target group are visually perceived as engaging in coordinated action. If so, biases concerning perceived threat, collective responsibility, and so on, may be more accessible, and thus more predictive of behavior, when these sorts of cues are present.

\section{How entitativity perception modulates accessibility}

I have been arguing that entitativity perception modulates the activation of the biases and stereotypes that implicit measures are designed to detect. But how does entitativity perception play this modulatory role? The notion of conceptual centrality may be helpful here.

According to prototype theory, concepts encode sets of weighted features. There are different views on what determines the weight of a feature, and how the weights determine membership in the relevant category. Nonetheless, it is widely agreed that some features are encoded as more "central" than others. If, for example, $f$ is relatively central, this means that other features depend on it more than it depends on them (Sloman et al., 1998; Thagard, 1989). Take Del Pinal and Spaulding's $(2018,100)$ example of the concept CHAIR. This concept encodes the features +HAS A BACK and +USED FOR SITTING. However, +USED FOR SITTING is more central than +HAS A BACK because the former explains the latter.

When it comes to your prototype of a given group, which features are central will depend on how you are thinking about the group. For example, if you construe it as an intimacy group, affiliative bonds will be among the most centrally encoded features, for these explain other features of the group, such as the close proximity of its members, as well as their friendliness towards one another. If, however, you construe the group as a task group, shared goals will be 
encoded as central, for these will explain group members' tendency towards coordinated action; joint attention; and so on.

Given that different features will be encoded as central for different types of groups, this can explain why the activation of implicit biases and stereotypes is constrained by perceived group type, as well as the group's degree of perceived entitativity.

For example, suppose you are in a context in which you are thinking of some racial group as an alliance. As a result, you perceive the group as high in dynamical entitativity, representing the sharing of certain goals as central. These representations thereby constrain your stereotypic expectations about members' behavior. For example, you expect to observe various forms of affiliative behavior; however, these expectations do not necessarily extend to irrelevant traits, such as being athletic or being tall. Thus, at the encoding stage, attention will go to those features that are cues for dynamical entitativity—recall that this is what Rutchick and colleagues (2008) found when they primed participants to think of the target group as an alliance. This attentional bias will result in associations between the target group and alliance-based traits: associations that are highly accessible when the relevant alliance cues are salient.

If, on the other hand, the context is such that you are thinking of this racial group as a social category, shared goals will not be represented as central. Which features you represent as central will depend on the degree to which you see its members as homogenous, i.e. the degree to which you perceive the group as being high in categorical entitativity. If you see the group as very homogenous then this raises the probability that you are representing its members as possessing some hidden trait (or essence) that explains the observed homogeneity. Given that this trait (or essence) is hidden, its nature will be somewhat opaque. For this reason, Medin and Ortony (1989) refer to the folk notion of an essence as a "placeholder notion": one believes that 
members of the given category possess an essence without knowing exactly what this essence consists in. This might explain why stereotyping is more promiscuous for social categories than it is for intimacy and task groups. For intimacy and task groups, the features that are represented as central are affiliative bonds and shared goals: this puts relatively tight constraints on stereotypic expectations. In contrast, for many social categories, the features that count as central are largely unknown. Thus, one's representations of the relevant dependencies will be somewhat schematic. Given the schematic nature of these representations, they will put relatively loose constraints on stereotypic expectations.

For example, if you are perceiving the given racial group as high in categorical entitativity, your stereotypic expectations may encompass various physical features, as well as various psycho-behavioral dispositions. Thus, at the encoding stage, attention will go to these sorts of features, not the sorts of features that are cues for dynamical entitativity perception (e.g. cues for shared attention; coordinated action; and so on). Moreover, the associations that are encoded as a result of this attentional bias will be more accessible when categorical entitativity cues are salient (i.e. when similarities between group members are salient). ${ }^{3}$

\section{Conclusion}

I have argued that entitativity perception modulates the activation of biases and stereotypes; and that by recognizing this fact, researchers will be able to design implicit measures with stronger validity and reliability.

Examining the relation between entitativity perception and implicit social cognition will likely have other payoffs as well. For example, some studies have found that moving in synchrony with

\footnotetext{
${ }^{3}$ There is, of course, much more to be said about the cognitive architecture underlying the accessibility of implicit biases and stereotypes. Various models could be applied to the general picture I have just sketched. For a brief discussion of relevant models, see Payne et al. $(2017,235)$.
} 
the members of an outgroup can reduce both implicit and explicit biases against them (see Atherton et al., 2019; Farmer \& Maister, 2017). Given that synchronous movement is a cue for dynamical entitativity perception, this is not surprising. In further examining the effectiveness of different interventions, future studies should be sensitive to the distinction between dynamical and categorical entitativity perception, including the various cues that trigger their activation across contexts.

Research on entitativity perception may also deepen our understanding of cross-cultural differences in the kinds of implicit biases and stereotypes that are encoded. Kurebayashi and colleagues (2012) found that Japanese individuals tend to engage in dynamical entitativity perception more than American individuals; whereas, American individuals tend engage in categorical entitativity perception more than Japanese individuals. If what I have argued is right, we should expect to find differences in the kinds of implicit biases and stereotypes that Japanese and American individuals tend to harbor.

Finally, I have been focusing on implicit measures of social cognition, but some of the studies mentioned above found that entitativity perception modulates the encoding and activation of explicit attitudes too (e.g., see Ip et al., 2006; Rutchick et al., 2008; Spencer-Rodgers et al., 2007). Thus, even though explicit attitudes are often thought to be more stable than implicit attitudes (e.g., see Gawronski et al., 2017), entitativity perception is likely an important source of context-sensitivity for both. 


\section{References}

Abelson, R. P., Dasgupta, N., Park, J., \& Banaji, M. R. (1998). Perceptions of the collective other. Personality and Social Psychology Review, 2, 243-250.

Ariely, D. (2001). Seeing sets: Representation by statistical properties. Psychological Science, 12, 157162.

Atherton, G., Sebanz, N., \& Cross, L. (2019). Imagine all the synchrony: The effects of actual and imagined synchronous walking on attitudes towards marginalised groups. PLoS ONE 14(5): e0216585. https://doi.org/10.1371/journal.pone.0216585

Bangerter, A. (2005). Entitativity of generations, age groups and cohorts as perceived by young adults. Swiss Journal of Psychology, 64(4), 273-280.

Bar-Anan, Y., \& Nosek, B. A. (2014). A comparative investigation of seven indirect attitude measures. Behavior Research Methods, 46, 668-688.

Barden, J., Maddux, W.W., Petty, R.E., \& Brewer, M.B. (2004). Contextual moderation of racial bias: The impact of social roles on controlled and automatically activated attitudes. Journal of Personality and Social Psychology, 87, 5-22.

Brewer, M. B., Hong, Y., \& Li, Q. (2004). Dynamic entitativity: Perceiving groups as actors. In V. Yzerbyt, C. M. Judd, \& O. Corneille (Eds.), The psychology of group perception: Contributions to the study of homogeneity, entitativity and essentialism (pp. 25-38). Philadelphia, PA: Psychology Press.

Brownstein, M., Madva, A., \& Gawronski, B. (2020). Understanding implicit bias: Putting the criticism into perspective. Pacific Philosophical Quarterly. https://doi.org/10.1111/papq.12302

Callahan, S. P., \& Ledgerwood, A. (2016). On the psychological function of flags and logos: Group identity symbols increase perceived entitativity. Journal of Personality and Social Psychology, 110(4), $528-550$.

Campbell, D. T. (1958). Common fate, similarity, and other indices of the status of aggregates of persons as social entities. Behavioral Science, 3, $14-25$. 
Cooley, E., \& Payne, B. K. (2017). Using groups to measure intergroup prejudice. Personality and Social Psychology Bulletin, 43(1), 46-59.

Crawford, M. T., Sherman, S. J., \& Hamilton, D. L. (2002). Perceived entitativity, stereotype formation, and the interchangeability of group members. Journal of Personality and Social Psychology, 83, 1076-1094.

De Houwer, J., \& Moors, A. (2010). Implicit measures: Similarities and differences. In B. Gawronski \& B. K. Payne (Eds.), Handbook of implicit social cognition: Measurement, theory, and applications (pp. 176-193). Guilford Press.

Del Pinal, G., \& Spaulding, S. (2018). Conceptual centrality and implicit bias. Mind and Language, 33, 95-111.

Enos, R. D. (2017). How segregation leads to racist voting by whites. Retrieved from: https://www.vox.com/the-big-idea/2017/11/28/16707438/social-geography-trump-rise-segregationpsychology-racism

Fazio, R. H., Jackson, J. R., Dunton, B. C., \& Williams, C. J. (1995). Variability in automatic activation as an unobtrusive measure of racial attitudes: A bona fide pipeline? Journal of Personality and Social Psychology, 69(6), 1013-1027.

Farmer, H., \& Maister, L. (2017). Putting ourselves in another's skin: Using the plasticity of selfperception to enhance empathy and decrease prejudice. Social Justice Research, 30(4), 323-354.

Fessler, D. M., \& Holbrook, C. (2016). Synchronized behaviour increases assessments of the formidability and cohesion of coalitions. Evolution and Human Behavior, 37(6), 502-509.

Forscher, P. S., Lai, C. K., Axt, J. R., Ebersole, C. R., Herman, M., Devine, P. G., \& Nosek, B. A. (2019). A meta-analysis of procedures to change implicit measures. Journal of Personality and Social Psychology: Attitudes and Social Cognition, 117(3), 522-559.

Gaertner, L., Iuzzini, J., \& O’Mara, E. M. (2008). When rejection by one fosters aggression against many: Multiple-victim aggression as a consequence of social rejection and perceived groupness. Journal of Experimental Social Psychology, 44, 958-970. 
Gawronski, B., Morrison, M., Phills, C., \& Galdi, S. (2017). Temporal stability of implicit and explicit measures: A longitudinal analysis,' Personality and Social Psychology Bulletin, 43(3), 300-312.

Gelman, S. A. (2003). The essential child: Origins of essentialism in everyday thought. Oxford University Press.

Greenwald, A., McGhee, D., \& Schwartz, J. (1998). Measuring individual differences in implicit cognition: The Implicit Association Test. Journal of Personality and Social Psychology, 74 (6), 14641480.

Gschwendner, T., Hofmann, W., \& Schmitt, M. (2008). Differential stability: The effects of acute and chronic construct accessibility on the temporal stability of the Implicit Association Test. Journal of Individual Differences, 29, 70-79.

Haberman, J., Harp, T., \& Whitney, D. (2009). Averaging facial expression over time. Journal of Vision, 9, $1-13$.

Hamilton, D. L. (2007). Understanding the complexities of group perception: Broadening the domain. European Journal of Social Psychology, 37, 1077-1101

Hamilton, D. L., Sherman, S. J., \& Castelli, L. (2002). A group by any other name-The role of entitativity in group perception. European Review of Social Psychology, 12(1), 139-166.

Haslam, N., Rothschild, L., \& Ernst, D. (2000). Essentialist beliefs about social categories. British Journal of Social Psychology, 39, 113-127.

Ip, G. W. M., Chiu, C. Y., \& Wan, C. (2006). Birds of a feather and birds flocking together: Physical versus behavioral cues may lead to trait- versus goal-based group perception. Journal of Personality and Social Psychology, 90, 368-381.

Jost, J. T. (2019). The IAT is dead, long live the IAT: Context-sensitive measures of implicit attitudes are indispensable to social and political psychology. Current Directions in Psychological Science, 28(1), 10-19. 
Kurebayashi, K., Hoffman, L. Ryan, C. S., Ryan, A., \& Murayama, A. (2012). Japanese and American perceptions of group entitativity and autonomy: A multilevel analysis. Journal of Cross-Cultural Psychology, 43(2), 349-364.

Lakens, D. (2010). Movement synchrony and perceived entitativity. Journal of Experimental Social Psychology, 46(5), 701-708.

Lakens, D., \& Stel, M. (2011). If they move in sync, they must feel in sync: Movement synchrony leads to attributions of rapport and entitativity. Social Cognition, 29(1), 1-14.

Lebrecht, S., Pierce, L. J., Tarr, M. J., \& Tanaka, J. W. (2009). Perceptual other-race training reduces implicit racial bias. PLoS One, 4(1), e4215. doi: 10.1371/journal.pone.0004215.

Leonard, D. J. (2019). They're a sorry bunch: Perceptions of outgroup entitativity shape the receipt of intergroup apology. Group Processes and Intergroup Relations, 22(4), 549-562.

Lickel, B., Hamilton, D. L., \& Sherman, S. J. (2001). Elements of a lay theory of groups: Types of groups, relational styles, and the perception of group entitativity. Personality and Social Psychology Review, 5(2), 129-140.

Lickel, B., Hamilton, D. L., Wieczokowska, G., Lewis, A., Sherman, S. J., \& Uhles, A. N. (2000). Varieties of groups and the perception of group entitativity. Journal of Personality and Social Psychology, 78, 223-246.

Lickel, B., Schmader, T., \& Hamilton, D. L. (2003). A case of collective responsibility: Who else was to blame for the Columbine High School shootings? Personality and Social Psychology Bulletin, 29, 194-204.

Magee, J. C., \& Tiedens, L. Z. (2006). Emotional ties that bind: the roles of valence and consistency of group emotion in inferences of cohesiveness and common fate. Personality and Social Psychology Bulletin, 32(12), 1703-1715.

Malle, B. F. (2010). The social and moral cognition of group agents. Journal of Law and Policy, 19, 95136. 
Medin, D. L., \& Ortony, A. (1989). Psychological essentialism. In S. Vosniadou \& A. Ortony (Eds.), Similarity and analogical reasoning (pp. 179-195). Cambridge University Press.

Newheiser, A-K, Sawaoka, T., \& Dovidio, J. F. (2012). Why do we punish groups? High entitativity promotes moral suspicion. Journal of Experimental Social Psychology, 48, 931-936.

Nosek, B., \& Banaji, M. (2001). The Go/No-Go Association Task. Social Cognition, 19(6): 625-666.

Oswald, F. L., Mitchell, G., Blanton, H., Jaccard, J., \& Tetlock, P. E. (2013). Predicting ethnic and racial discrimination: A meta-analysis of IAT criterion studies. Journal of Personality and Social Psychology, 105, 171-192

Payne, B. (2001). Prejudice and perception: The role of automatic and controlled processes in misperceiving a weapon. Journal of Personality and Social Psychology, 81(2): 181-192.

Payne, B. (2009). Attitude misattribution: Implications for attitude measurement and the implicit-explicit relationship. In R. Petty, R. Fazio, \& P. Brinol (Eds.), Attitudes: Insights from the new wave of implicit measures (pp. 459-484). Hillsdale, NJ: Erlbaum.

Payne, B. K., Vuletich, H. A. \& Lundberg, K. B. (2017). Flipping the script on implicit bias research with the bias of crowds. Psychological Inquiry, 28(4), 306-311.

Phillips, B. (2020). The roots of racial categorization. Review of Philosophy and Psychology. DOI: https://doi.org/10.1007/s13164-021-00525-w

Phillips, L. T., Slepian, M. L., \& Hughes, B. L. (2018). Perceiving groups: The people perception of diversity and hierarchy. Journal of Personality and Social Psychology, 114(5), 766-785.

Phillips, L. T., Weisbuch, M., \& Ambady, N. (2014). People perception: Social vision of groups and consequences for organizing and interacting. Research in Organizational Behavior, 34, 101-127.

Pietraszewski, D. (2016). Priming race: Does the mind inhibit categorization by race at encoding or recall? Social Psychological and Personality Science, 7(1), 85-91.

Rhodes, M. (2012). Naïve theories of social groups. Child Development, 83(6), 1900-1916. 
Rothbart, M., \& Taylor, M. (1992). Category labels and social reality: Do we view social categories as natural kinds? In G. R. Semin \& K. Fiedler (Eds.), Language, interaction and social cognition (pp. 11-36). London: Sage.

Rutchick, A. M., Hamilton, D. L., \& Sack, J. D. (2008). Antecedents of entitativity in categorically and dynamically construed groups. European Journal of Social Psychology, 38, 905-921.

Sherman, S. J., Castelli, L., \& Hamilton, D. L. (2002). The spontaneous use of a group typology as an organizing principle in memory. Journal of Personality and Social Psychology, 82, 328-342.

Shutts, K., Roben, C., \& Spelke, E. (2013). Children's use of social categories in thinking about people and social relationships. Journal of Cognition and Development, 14(1), 35-62.

Singal, J. (2017). Psychology's favorite tool for measuring racism isn't up to the job. New York Magazine. Retrieved from: https://www.thecut.com/2017/01/psychologys-racism-measuring-tool-isntup-to-the-job.html

Sloman, S. A., Love, B. C. \& Ahn, W. K. (1998). Feature centrality and conceptual coherence. Cognitive Science, 22, 189-228.

Spencer-Rodgers, J., Hamilton, D. L., \& Sherman, S. J. (2007). The central role of entitativity in stereotypes of social categories and task groups. Journal of Personality and Social Psychology, 92, $369-388$.

Susskind, J., Maurer, K., Thakkar, V., Hamilton, D. L., \& Sherman, S. J. (1999). Perceiving individuals and groups: Expectancies, dispositional inferences, and causal attributions. Journal of Personality and Social Psychology, 76, 181-191.

Tanibe, T., Hashimoto, T., Tomabechi, T., Masamoto, T., \& Karasawa, K. (2019). Attributing mind to groups and their members on two dimensions. Frontiers in Psychology, 10(840). DOI:

\subsection{9/fpsyg.2019.00840}

Thagard, P. (1989). Explanatory coherence. Behavioral and Brain Sciences, 12, 435-467.

Thorpe, S., Fize, D., \& Marlot, C. (1996). Speed of processing in the human visual system. Nature, 381, $520-522$. 
Wilder, D., \& Simon, A. F. (1998). Categorical and dynamic groups: Implication for social perception and intergroup behavior. In C. Sedikides, J. Schopler, \& C. A. Insko (Eds.), Intergroup cognition and intergroup behavior (pp. 27-44). Mahwah, NJ: Erlbaum.

Wilson, S., \& Gos, C. (2019). Perceiving social cohesion: Movement synchrony and task demands both matter. Perception, 48(4), 316-329.

Winegard, B., \& Deaner, R. O. (2010). The evolutionary significance of Red Sox nation: sport fandom as a byproduct of coalitional psychology. Evolutionary Psychology, 8(3), 432-446.

Yzerbyt, V. Y., Rogier, A., \& Fiske, S. (1998). Group entitativity and social attribution: On translating situational constraints into stereotypes. Personality and Social Psychology Bulletin, 24, 1090-1104.

Yzerbyt, V. Y., Rocher, S., \& Schadron, G. (1997). Stereotypes as explanations: A subjective essentialistic view of group perception. In R. Spears, P. Oakes, N. Ellemers, \& A. Haslam (Eds.), The psychology of stereotyping and group life (pp. 20-50). London: Basil Blackwell. 